\title{
Design and Synthesis of the Next Generation of Crown Ethers for Waste Separations: An Interlaboratory Comprehensive Proposal
}

\author{
Oak Ridge National Laboratory \\ September 1997 \\ Progress Report
}

\section{Principal Investigator}

Bruce A. Moyer

(423) 574-6718 (Phone)

(423) 574-4939 (Fax)

moyerba@ornl.gov

Oak Ridge National Laboratory

Bldg. 4500S, MS-6119, P. 0. Box 2008

Oak Ridge, TN 37831-6119

\section{Co-investigators}

Mark L. Dietz

(708) 252-3647 (Phone)

(708) 252-7501 (Fax)

markdietz@qmgate.anl.gov

Argonne National Laboratory

Bldg. 200, 9700 South Cass Ave.

Argonne, IL 60439-4831

Benjamin P. Hay

(509) 372-6239 (Phone)

(509) 372-6328 (Fax)

bp_hay@gate.pnl.gov

Battelle Pacific Northwest Nat'1 Laboratory

Battelle Blvd., K6-822

Richland, WA 99352

Spiro D. Alexandratos

(423) 974-3399 (Phone)

(423) 974-3454 (Fax)

alexsd@utk.edu

University of Tennessee

Knoxviile, TN 37996

\section{Contributors}
R. Chiarizia, ANL
D. A. Dixon, PNNL
J. C. Bryan, ORNL
J. B. Nicholas, PNNL
T. J. Haverlock, ORNL
L. Yang, PNNL
R. A. Sachleben, ORNL 


\section{Research Objective}

The purpose of this task is to undertake the design, synthesis, and characterization of the next generation of crown ethers for metal-ion separations applicable to the U.S. Department of Energy's (DOE's) environmental needs. The general target problem is the removal of alkali and alkaline-earth metal contaminants from certain environmental and waste streams. Although not a radioactivity hazard, $\mathrm{Li}^{+}$ions leaching from burial sites containing more than 12 metric tons of lithium compounds contaminate the groundwater at the Oak Ridge Y-12 Plant and have raised noncompliance concerns because of the resultant toxicity to aquatic biota.

A more highly visible problem has been treatment of high-level wastes stored in underground tanks at Oak Ridge National Laboratory (ORNL), Idaho National Engineering and Environmental Laboratory, and especially the Hanford Site. The fission products ${ }^{90} \mathrm{Sr}$ and ${ }^{37} \mathrm{Cs}$ have been explicitly targeted for removal by the following DOE programs: the Office of Environmental Management, the Office of Science and Technology, the Tank Waste Remediation System, the Tanks Focus Area, and the Efficient Separations and Processing Cross-Cutting Program. These seemingly ubiquitous fission products also appear in soil and groundwater at numerous DOE sites. In addition, radium has recently been named as a target contaminant at the Niagara Falls Storage Site.

Unfortunately, the separations technologies needed to address these problems either do not exist or exhibit substantial deficiencies. Established techniques such as solvent extraction and ion exchange certainly have a strong role to play, especially as enhanced with the use of the new highly selective metal-ion hosts such as crown ethers and calixarenes. Recently applied results in the United States, France, Russia, and elsewhere have demonstrated the effective performance of crown ethers under realistic or actual process conditions for the removal of alkali and alkaline-earth metal ions from waste, including high-levelnuclear waste.

From these results, one can readily appreciate how improvements in selectivity and extraction strength can immediately translate to improvements and cost savings in existing technologies. One can also envision extending crown-ether-based chemistry to solve other problems, such as groundwater remediation. Achieving these ends will require the design and synthesis of new crown compounds with "next-generation" capabilities, which will in turn require efforts to build upon the current base of fundamental knowledge pertaining to host-guest chemistry, solvent extraction, and ion exchange.

\section{Research Statement}

In view of the needs and aims outlined above, this task combines three interdependent projects dealing with (1) molecular mechanics and ligand design, (2) solvent extraction of univalent and divalent metal ions, and (3) polymer-immobilized crown ethers. The partner laboratories at ORNL, Pacific Northwest National Laboratory, Argonne National Laboratory, and the University of Tennessee, Knoxville (UTK), have world-recognized programs in the area of crown ethers, solvent extraction, and ion exchange. Their cooperation under this task represents an unusual and effective combination of unique resources.

The general approach entails the use of computational methods to predict properties and strategies for testing the resulting predictions. State-of-the-art molecular-mechanics calculations are being used as a tool in the design of new crown ethers for the selective extraction of alkali 
and alkaline-earth metal ions. Synthetic investigations are focusing on the development of efficient preparations of crown ethers designed to possess either lipophilicity for solvent extraction or polymerizable functionalities for immobilization in resin beads.

Key questions deal with the function of cation-exchange (often termed proton-ionizable) groups and whether the ability to fully satisfy both the coordination and charge-neutralization requirements of a given metal ion can be incorporated into crown-containing compounds, solvents, or materials. Relationships between the function of crown ethers in solvent extraction and their function in a polymer environment will be revealed upon immobilization in polymerization reactions. At the same time, how such factors relate to selectivity for both charge-dense and charge-diffuse spherical ions will be addressed for alkali and alkaline-earth metals. Representative ions include $\mathrm{Li}^{+}, \mathrm{Cs}^{+}, \mathrm{Sr}^{2+}$, and $\mathrm{Ra}^{2+}$, each of which poses an environmental problem at a given DOE site.

\section{Research Progress}

\section{Molecular Mechanics and Ligand Design}

This report documents molecular-modeling activities toward the rational design of the next generation of crown ethers for waste separations. Progress was made in four areas: MM3 computer model parameter development for pi-cation interactions, MM3 model development for phosphorous oxyanions, structure-function relationships for multidentate ether ligands, and model application in an attempt to optimize cesium/potassium selectivity through structural modification of a calix[4]arene-crown-6 ligand.

An MM3 parameter set has been completed to treat the pi-cation interactions between the alkali cations and uncharged arenes. The development of this parameter set involved electronic structure calculations on cation-benzene complexes as well as empirical fitting to crystal structures containing alkali metal cation-arene binding. Comparison of calculated and observed M-Csp2 distances in 18 crystal structures demonstrates the model is accurate to within $\pm 0.2 \mathrm{~A}$.

The development of an MM3 parameter set for dialkylphosphates, dialkylphosphonates, and dialkylphosphinates and their complexes with the alkali and alkaline-earth cations is in progress. A series of density-functional-theory calculations has been completed to obtain ground-state geometries and potential-energy surfaces for the dimethyl analogues of these ligands and their complexes with $\mathrm{Li}^{+}, \mathrm{K}^{+}, \mathrm{Mg}^{+}$, and $\mathrm{Ca}^{2+}$ ions. A set of $\mathrm{MM} 3$ parameters for the dialkylphosphinate functional group has been completed. A set of MM3 parameters for the dialkylphosphonate and dialkylphosphate functional groups will be completed by the end of the fiscal year.

Molecular-mechanics analysis of binding site organization in a series of anisole spherand derivatives has led to the first structure-function relationship for this class of compounds. The extended MM3 model has also been applied in the analysis of the effect of structural modification on binding-site complementarity for cesium and potassium ions in a series of six derivatives of a calix[4]-crown-6 ligand. 


\section{Solvent Extraction of Univalent and Divalent Metal Ions}

Divalent Metal Ions. In an effort to develop guidelines for the rational design of synergistic extraction systems for alkali and alkaline-earth cations using macrocyclic polyethers (crown ethers), we have been examining the extraction of selected cations into toluene by various organophosphorus acids and crown ethers, both alone and in combination. Although synergistic effects involving mixtures of crown ethers and various organophilic acid anions are well known, few of the previously described studies have taken a systematic approach to the elucidation of the factors responsible for the observed synergism (or antagonism). In the present work, in contrast, structurally well-defined macrocycles and organophilic anions are employed, thereby providing a means of determining the influence of the crown ether stereochemistry and anion structure on the observed synergistic effects. In this way, the specific steric requirements for effective synergism can be defined.

Our initial work has focused on the use of bis(n-octyl) phosphoric acid (abbreviated HDOP) and bis(2-ethylhexyl) phosphoric acid (abbreviated HDEHP) as the organophilic counterions, in combination with the cis-syn-cis (A) and cis-anti-cis (B) isomers of dicyclohexano- 18-crown-6 (DCH18C6). The synergism between HDOP and DCH18C6-A and DCH18C6-B has been investigated in detail using solvent extraction, infrared spectroscopy, and vapor-phase osmometry (VPO). Extraction results for calcium, strontium, and barium indicate that isomer B generally yields weaker synergistic effects than does isomer A under the same conditions. In all cases where synergism is observed, continuous-variation studies indicate that the ratio of HDOP to DCH 18C6 in the, complex is 4: 1 (2 HDOP dimers to 1 crown ether molecule). Crown ether concentration dependencies at fixed HDOP concentration exhibit the expected unit slope at low crown concentrations but significant negative deviation at higher concentrations, a likely result of the solubility of the metal crown complex in the aqueous phase.

VP0 indicates that, as expected, HDOP exists as a dimer and DCH18C6 (A or B) as a monomer under the experimental conditions. Moreover, the presence of small concentrations of metal ion has no appreciable effect on the aggregation behavior. Mixtures of HDOP and DCH 18C6 exhibit behavior consistent with an absence of interaction between the two species. This is confirmed by the results of infrared (IR) studies, which show that the JR spectra of HDOP-DCH 18C6 mixtures are essentially the sum of the component spectra.

Work to model the extraction equilibria for this system is in progress, as are IR and VP0 studies of the branched analog of HDOP (i.e., HDEHP). Extraction studies using other DCH18C6 isomers and/or a third, highly branched organophosphorus acid [i.e., bis(diisobutylmethyl)phosphoric acid] will commence shortly.

Univalent Metal Ions. Synthetic efforts toward the development of improved cesium-selective extractants based on calix[4]arene-crown ethers have taken two tracks. Initially, quantities of bis(t-octylbenzo-crown-6)-calix[4]arene (BOBC6calix[4]) sufficient for detailed equilibrium modeling studies were synthesized. Subsequently, a campaign to develop syntheses of calix[4]-monocrown- ethers bearing short bridging groups between two of the calixarene phenols was undertaken. Initial efforts to bridge two phenol groups regiochemically at the 1,3 positions by four- or five-carbon ether or ester linkages failed to provide the desired bridged calixarenes. Therefore, an alternative approach whereby two substituents located in the 1,3 positions will be linked is being investigated.

The BOBC6calix[4] was subjected to an analysis of its selectivity behavior toward extraction of cesium vs potassium. This investigation was motivated in particular by the significant 
concentrations of potassium in certain alkaline tank wastes, resulting in loading of the extractant by potassium and reduced effectiveness in removal of cesium. The results reveal a good selectivity for cesium vs potassium overall, yielding values of the selectivity ratio $S_{\mathrm{CsK}}$ (i.e., ratio of distribution coefficients) in the range 100-600. Within that range, the value of $\mathrm{S}_{\mathrm{csk}}$ depends on the diluent, increasing as the electron-pair donor strength and polarity of the diluent decrease. Selectivity also increases with decreasing overall loading of the calixarene, suggesting a deleterious effect of ion-pairing involving the nitrate anion. Preliminary equilibrium modeling with the program SXLSQI has been initiated and shows consistency in general with the types of speciation normally expected based on predominant 1: 1 complexation of the metal by the extractant and partial ion-pairing with nitrate anion. Good evidence for 2: 1 metal:calixarene species exists for both $\mathrm{K}$ and $\mathrm{Cs}$, but this requires high-loading conditions, which for cesium at trace concentration in waste would be unlikely.

\section{Polymer-Immobilized Crown Ethers}

A quantity (50 g) of 6-methyleno, octamethyl-14-crown-4 was provided to UTK for studies of the copolymerization of t-alkyl- 14-crown4 ethers into polystyrene/polyacrylonitrile copolymers. Subsequently, two new crown ethers were synthesized, 6-keto, octamethyl-14-crown-4 and 6-hydroxymethyl, octamethyl-14-crown-4 (about $5 \mathrm{~g}$ each), for studies of appending t-alkyl-14-crown-4 ethers onto intact polystyrene/polyvinylbenzyl chloride polymers.

An extensive series of experiments has been completed to determine whether it is possible to copolymerize the vinylidene crown ether (14C4) with other monomers. We have studied another sterically hindered monomer, vinylidene- 1, 1-diphosphonic acid, extensively and have learned that it can be copolymerized with monomers such as acrylonitrile, even though it shows no tendency to homopolymerize. Copolymers studied in the present case were acrylonitrile, vinylbenzyl chloride, divinylbenzene, methyl methacrylate, and ethyl acrylate. The free radical initiator was benzoyl peroxide, in concentrations from $0.5 \%$ to $8 \%$ (by weight, relative to the entire monomer solution). It may be concluded that over a wide range of comonomer concentrations and polymerization conditions, there is negligible incorporation of the crown into the polymers formed. In all cases, elemental analyses and Fourier transform IR spectra are consistent with the absence of crown ether in the final polymers, though a low degree of lithium tetraphenylborate uptake (about $0.1 \mathrm{meq} / \mathrm{g}$ ) was noted. As a result, we have decided to immobilize the crown onto appropriately substituted polystyrene beads. The first attempt will be through the ketone derivative of the crown; therefore, we have devised a method to place amine ligands on polystyrene with potassium phthalimide. The synthesis of the Schiff base will be attempted in the next set of experiments.

\section{Summary of Accomplishments}

- Toward designing new calixarene-type extractants selective for cesium ion, an MM3 parameter set has been completed to treat pi-cation interactions between alkali cations and uncharged arenes.

- Molecular-mechanics analysis of binding site organization in a series of anisole spherand derivatives has led to the first structure-function relationship for this class of compounds. The 
extended MM3 model has also elucidated selectivity for cesium vs. potassium ions in a series of six calix[4]-crown-6 ligands.

- The extraction of the alkaline earth metals calcium, strontium, and barium by cis-syn-cis (A) and cis-anti-cis (B) isomers of dicyclohexano- 18-crown-6 (DCH18C6) was shown to be strongly enhanced by selected dialkyl phosphoric acids.

- The selectivity for cesium vs potassium for the alkane-soluble cesium extractant bis(t-octylbenzo-crown-6)-calix[4]arene was determined and shown to be sensitive to the diluent and concentration variables.

- Three 14-crown-4 ethers were synthesized and given to UTK for incorporation into polymers, either by co-polymerization or substitution onto derivatized polystyrene sm $\Omega$.

\section{Papers and Abstracts}

Haverlock, T. J,. P. V. Bonnesen, R. A. Sachleben, and B. A. Moyer. 1997. "Cs/K Selectivity of a Lipophilic CaIix[4]arene-Crown-6 Extractant in Liquid-Liquid Separations from Nitrate Media." Presented at the 214th American Chemical Society National Meeting, Las Vegas, Nev., September 7-11,1997

Hay, B. P. "A Molecular Mechanics Method for Predicting the Influence of Ligand Structure on Metal Ion Binding Affinity." Submitted to Progress in Metal Ion Separation and Preconcentration, ACS Symposium Series.

Yang, L., D. A. Dixon, and B. P. Hay. 1997. "MM3 Calculations on Alkali Cation Benzene Complexes." Presented at the International Conference on Structural and Mechanistic Organic Chemistry, Athens, Ga., June 6,1997.

Yang, L., D. A. Dixon, and B. P. Hay. 1997. "Molecular Mechanics (MM3) Calculations on Calixarene Complexes with Alkali Metal Cations: The Importance of $\pi$-Cation Interactions." Presented at the 214th American Chemical Society Meeting, Las Vegas, Nev., September 7-1 1, 1997. 